\title{
Association of hysterectomy and invasive epithelial ovarian and tubal cancer: A cohort study within UKCTOCS
} \author{
Alexandra Gentry-Maharaj ${ }^{1}$ \\ ${ }^{1}$ University College London \\ ${ }^{2}$ Imperial College Healthcare NHS Trust \\ ${ }^{3}$ University Hospital of Wales \\ ${ }^{4}$ Queen Mary University of London \\ ${ }^{5}$ Queen Alexandra Hospital \\ ${ }^{6}$ Create Health Clinics \\ ${ }^{7}$ Barts Health NHS Trust \\ ${ }^{8}$ University of New South Wales
}

Julie Taylor ${ }^{1}$, Matthew Burnell ${ }^{1}$, Andy Ryan $^{1}$, Chloe Karpinskyj ${ }^{1}$, Jatinderpal Kalsi ${ }^{1}$, Henry Taylor ${ }^{2}$, Sophia Apostolidou ${ }^{1}$, Aarti Sharma ${ }^{3}$, Ranjit Manchanda ${ }^{4}$, Robert Woolas ${ }^{5}$, Stuart Campbell ${ }^{6}$, Mahesh Parmar ${ }^{1}$, Naveena Singh ${ }^{7}$, Ian Jacobs ${ }^{8}$, Usha Menon ${ }^{1}$, and

June 30, 2021

\begin{abstract}
Objective: To investigate the association between hysterectomy with conservation of one or both adnexa and ovarian and tubal cancer. Design: Prospective cohort study. Setting: 13 NHS Trusts in England, Wales and Northern Ireland. Population: 202,506 postmenopausal women recruited between 2001-2005 to the UK Collaborative Trial of Ovarian Cancer Screening (UKCTOCS) and followed up until 31 December 2014. Methods: Multiple sources (questionnaires, hospital notes, Hospital Episodes Statistics, national cancer/death registries, ultrasound reports) were used to obtain accurate data on hysterectomy (with conservation of one or both adnexa) and outcomes censored at bilateral oophorectomy, death, ovarian/tubal cancer diagnosis, loss to followup or 31 December 2014. Cox proportional hazards regression models were used to assess the association. Main outcome measures: Invasive epithelial ovarian and tubal cancer (WHO 2014) on independent outcome review. Results: Hysterectomy with conservation of one or both adnexa was reported in 41,912 (20.7\%; 41,912/202,506) women. Median follow up was 11.1 years (IQR 9.96-12.04), totalling >2.17million women-years. Among women who had undergone hysterectomy, 0.55\% (231/41912) were diagnosed with ovarian/tubal cancer, compared with $0.59 \%(945 / 160594)$ of those with intact uterus. Multivariable analysis showed no evidence of an association between hysterectomy and invasive epithelial ovarian/tubal cancer $(\mathrm{RR}=0.98$, 95\% CI 0.85-1.13, $\mathrm{p}=0.765)$. Conclusions: This large cohort study provides further independent validation that hysterectomy is not associated with alteration of invasive epithelial ovarian and tubal cancer risk. This data is important both for clinical counselling and for refining risk prediction models.
\end{abstract}

\section{Hosted file}

Taylor et al_Hysterectomy and OC_Manuscript_BJOG_Submitted_Updated_20210623.docx available at https://authorea.com/users/422860/articles/528487-association-of-hysterectomy-andinvasive-epithelial-ovarian-and-tubal-cancer-a-cohort-study-within-ukctocs 